\title{
Evaluation of clinical, etiological and antimicrobial resistance profile of pediatric urinary tract infections in a secondary health care centre
}

\author{
Gökce Celep ${ }^{1}$, Hüseyin Burak Özçelik²
}

1. Amasya University, Faculty of Medicine, Department of Pediatrics, Amasya, Turkey.

2. Amasya University, Sabuncuoğlu Şerefeddin Education and Research Hospital, Microbiology Laboratuary, Amasya, Turkey.

\section{Author emails:}

Gökce Celep: ORCID ID: 0000-0001-6250-5096, E mail: gokce4celep@yahoo.com; Hüseyin Burak Özçelik: ORCID ID: 0000-0003-1872-2071, E mail address: h.burakozcelik@gmail.com

\begin{abstract}
Background: Urinary tract infections are common during childhood. The etiologic agents and empirical antibiotics may vary due to age and geographic area.

Objectives: This study was designed to investigate the urinary tract infection pathogens, their antibiotic resistance profile and risk factors in a sample of well-child population.

Materials and Methods: This retrospective study was conducted in the pediatric clinics of a secondary health-care centre in a one-year period. The source of data was hospital and laboratory records. Toilet trained children and adolescents aged between 5-17 years old with positive urine culture were enrolled into the study. Microbiological studies were conducted according to international guidelines.

Results: During the study 3640 urine samples were analyzed and 342(9.4\%) had significant growth. Gram negative enterobacteria were the most common infectious agents. Antibiotic susceptibility tests showed low cephalosporine resistance unless ESBL was positive. Multi drug resistance was remarkable. Extended beta lactamase resistance rate was 17\%. Previous history of antibiotic use before the present administration was the only significant risk factor for ESBL positivity.

Conclusion: Treating urinary tract infections may become an emerging problem soon. Unless there are risk factors, cephalosporines are good options, but if so nitrofurantoin or carbapanems should be preferred for treatment in this population. Keywords: Urinary tract infections, antibiotics, susceptibility.

DOI: https://dx.doi.org/10.4314/ahs.v21i2.10

Cite as: Celep G, Özselik HB. Evaluation of clinical, etiological and antimicrobial resistance profile of pediatric urinary tract infections in a secondary health care centre. Afri Health Sci. 2021;21(2). 557-565. bttps:// dx.doi.org/10.4314/abs.v21i2.10
\end{abstract}

\section{Introduction}

Urinary tract infections (UTI) are common febrile diseases during childhood. In fever of unknown origin, UTI must be considered as a differential diagnosis. 1 The prevalence of pediatric UTI was reported as $2-20 \%$ worldwide ${ }^{1,2}$. The infection has no specific signs and symptoms that is why clinical suspicion and laboratory tests are important. ${ }^{3}$ After the first episode, recurrent
Corresponding author:
Gökce Celep,
Amasya University,
Faculty of Medicine,
Department of Pediatrics, Amasya/ Turkey
Tel:+90 5326082577
Email: gokce4celep@yahoo.com

infections can occur within 6-12 months ${ }^{4}$. The infection may be community acquired or as a complication related to hospitalization by increasing health care costs and morbidity rate ${ }^{5}$. Timely diagnosis and effective antibiotic therapy can prevent the complications of UTI. Infection itself may cause morbidity and mortality due to pyelonephritis and septicaemia. Also, renal scarring, chronic renal failure, hypertension may be the long term complications $^{6-8}$. The etiologic agents are determined by culture studies, but empirical antibiotics according to age and geographic data are often prescribed before the culture results and antibiogram reports ${ }^{9}$. However increasing antibiotic resistance of urinary system pathogens is a global problem. Inappropriate and widespread use of antibiotics has led to multidrug resistant (MDR) pathoens ${ }^{10}$. The right choice and effective dose are also 
important to prevent nephrotoxicity ${ }^{11}$. The antimicrobial susceptibility of the microorganisms and risk factors causing multi drug resistance may vary regionally ${ }^{9,12}$ Bacteria gain resistance to beta-lactam antibiotics (synthetic penicillins, cephalosporins, aztreonam) and this makes the therapy process complicated with poor outcome and/or application of expensive, broad spectrum antibiotics such as carbapenems ${ }^{13,14}$. Rising carbapenem resistance is another problem ${ }^{15}$. The rise in extendedspectrum -beta lactamase (ESBL) producing bacteria in UTI is a therapeutic problem in children since the treatment options are limited and not suitable for outpatient settings ${ }^{16}$. Infancy, uroprophylaxis, recent antibiotic therapy, recurrent UTI, male gender have been reported as risk factors for UTI with ESBL-producing organisms ${ }^{17}$. ESBL producing organisms present an ever-growing burden not only for hospitalized patients, but for community settings as well ${ }^{18,19}$.

The infectious agents and their antibiotic resistance patterns may vary between populations and regions. This study was designed to investigate the urinary tract infection pathogens, their antibiotic resistance profile and risk factors related with these parametres in a sample of previously healthy pediatric population who were admitted to the pediatric clinics of a secondary health care center. We aimed to emphasize risk factors for ESBL $(+)$ uropathogens and recommend therapeutic choices available for children suffering from UTI.

\section{Material and methods}

Study design and data collection: This retrospective study was conducted between $1^{\text {st }}$ April, 2016 and $31^{\text {st }}$ March, 2017 in the pediatric clinics of a secondary health- care centre in the middle-northern Turkey. The source of data was hospital and laboratory records. All toilet trained patients aged between 5 and 17 years old with positive urine culture, either admitted to pediatric outpatient clinics or hospitalized, were enrolled into the study. Children having no toilet training were excluded as urine bag samples or collecting urine via catheterization was not suitable for standardization. Patients with risk factors for recurrent UTI such as urogenital anomalies or neuromuscular problems related with non-optimal urine drainage were also excluded. Only one positive culture per patient was included in the study and repeated cultures from the same patient at the same time were excluded from the analysis. All urine specimens were obtained by midstream clean-catch method in toilet-trained children. ${ }^{19}$ Bag urine samples were not taken in consideration. Age, gender, hospital admission within 3 months, hospitalization status at the time of positive sample, season, the microorganisms in the urine culture, their antibiotic susceptibility profile, ESBL status, prescribed antimicrobial drugs 3 months before the positive urine specimen were noted through electronic hospital record system (Sisoft HBYS ${ }^{\circledR}$ ). "Laboratory UTI" was defined as "positive urine dipstick for nitrites and/or leukocyte count $>5$ / HPF. "Positive urine culture" was defined as "monomicrobial culture $\geq 100,000$ colony-forming units $[\mathrm{CFU}] / \mathrm{mL}$ for midstream and catheter urine". UTIs were classified as community acquired or healthcare associated due to patients' history. Community-acquired UTI (CA-UTI) was defined as a UTI episode in which, at the time the index urine sample was submitted, the patient was not hospitalized and had not been previously hospitalized during the preceding 30 days. Healthcare associated infection (HAI) was defined as "infections that patients acquire during the course of receiving healthcare treatment" ${ }^{5,14}$.

Microbiological procedures: The sterile mid-stream urine specimens were planted on 5\% blood agar and Eosin Methylene-blue Lactose Sucrose Agar (EMB) by semi-quantitative planting method with calibrated loop (0.001 ml). Planted plates were incubated aerobically at $37^{\circ} \mathrm{C}$ for $18-24$ hours. Samples having significant growth (that is $\geq 10^{5}$ colony forming units $(\mathrm{CFU} / \mathrm{ml}$ )) were processed for further identification and susceptibility testing via VITEK 2 Compact (Biomerieux-France) system according to the manufacturer's instructions. Antimicrobial susceptibilities and ESBL production were determined due to the European Committee on Antimicrobial Susceptibility Testing guidelines ${ }^{20}$. The microorganisms reported as ESBL ( + ) by VITEK went through confirmation tests by double disc synergy method that tests the susceptibility of the strain against amoxycillin and amoxycillin / clavulanic acid. The strain that showed increased inhibition zone with the combination disc on Mueller- Hinton agar plate was considered to have ESBL. E.coli ATCC 25922 was used as control test.

Statistical analyses: The analyses were performed using SPSS version 15 (SPSS, Inc., Chicago, IL, USA). The data were presented as frequencies, medians and minimum-maximum, range or mean \pm SD by descriptive statistics, when indicated. Cross- tabs with chi-square test $\left(\chi^{2}\right)$ and $z$-test were used to identify statistically significant differences between groups at $95 \%$ confidence. Probability factor (p) less than 0.05 was regarded as statistically significant. 
Ethics statement: The Ethical Committee of Hitit University approved this study (approval number: 2019103)

\section{Results}

During the study period (1 ${ }^{\text {st }}$ April, 2016-31 ${ }^{\text {st }}$ March, 2017), 8381 urine samples were accepted to the microbiology laboratory for culture tests and 3640 samples were suitable for this study. The samples belonging to children under five years old and having no toilet training $(n=4710)$ or patients with risk factors for recurrent UTI such as urogenital anomalies or neuromuscular problems related with non-optimal urine drainage $(\mathrm{n}=31)$ were excluded. Among 3640 UTI suspected samples, $342(9.4 \%)$ had significant growth. Of the 342 isolates $33(9.6 \%)$ belonged to males and 309 (90.4\%) to females and the mean age of the study group was $9.04 \pm 3.46$ years old. Eighty-one (23.7\%) of the samples were obtained in winter, 160 in autumn and spring equally and the rest $(\mathrm{n}=101 ; 29.5 \%)$ in summer. Only 17 $(4.9 \%)$ samples belonged to hospitalized patients (HAI) and the rest belonged to the patients of outpatient clinics (community acquired infections). Most of the specimens were from the pediatric emergency service $(\mathrm{n}=189 ; \% 55.3)$. Abdominal pain $(\mathrm{n}=89 ; 26.1 \%)$, dysuria $(n=45 ; 13.2 \%)$, nausea and vomiting $(n=12.9 \%)$ were the most common symptoms during administration (Table 1).

Table 1: Clinical features of the study population (page 7)

\begin{tabular}{|c|c|c|}
\hline Age & $9.04 \pm 3.46(5-17)$ years & \\
\hline \multicolumn{3}{|l|}{ Gender } \\
\hline Girls & 2538 & $69.7 \%$ \\
\hline Boys & 1102 & $30.3 \%$ \\
\hline \multicolumn{3}{|l|}{ Season } \\
\hline Autumn & 80 & $23.4 \%$ \\
\hline Winter & 81 & $23.7 \%$ \\
\hline Spring & 80 & $23.4 \%$ \\
\hline Summer & 101 & $29.5 \%$ \\
\hline \multicolumn{3}{|l|}{ Service } \\
\hline Pediatric Emergency Service & 189 & $55.3 \%$ \\
\hline Pediatrics & 105 & $30.7 \%$ \\
\hline Pediatric Surgery & 48 & $14 \%$ \\
\hline \multicolumn{3}{|l|}{ Patient setting } \\
\hline Outpatient clinics & 325 & $95 \%$ \\
\hline Hospitalized & 17 & $5 \%$ \\
\hline \multicolumn{3}{|l|}{ Reason for administration } \\
\hline Abdominal pain & \begin{tabular}{|l|}
89 \\
\end{tabular} & $26.1 \%$ \\
\hline Dysuria & 45 & $13.2 \%$ \\
\hline Nausea \pm vomiting & 24 & $7.0 \%$ \\
\hline Fever & 20 & $5.8 \%$ \\
\hline Enuresis & 7 & $2.0 \%$ \\
\hline Anorexia & 2 & $0.6 \%$ \\
\hline \multicolumn{3}{|l|}{ Dipstick test } \\
\hline Compatible with UTI & 212 & $62 \%$ \\
\hline Non- compatible with UTI & 54 & $15.8 \%$ \\
\hline \multicolumn{3}{|l|}{ Patient history } \\
\hline $\begin{array}{l}\text { Hospital administration before } \\
\text { the index positive urine culture }\end{array}$ & \multirow{3}{*}{\multicolumn{2}{|c|}{$1.89 \pm 2.29$ (median: 1$)$}} \\
\hline $\begin{array}{l}\text { Hospitalization before the index } \\
\text { positive urine culture }\end{array}$ & & \\
\hline Antibiotics (box per patient) & & \\
\hline
\end{tabular}


Among the study group, 218 patients were admitted to the hospital at least once (range: $0-13$ ) and 10 were hospitalized three months before the positive urine culture result. According to the accessed records 79 had respiratory tract infections, 53 had UTI and 26 acute diarrhea and vomiting. Approximately half of the patients $(\mathrm{n}=156 ; 45.6 \%)$ had received several antibiotics at different times within the preceding three months. Amoxicillin clavulanic acid ( $\mathrm{n}=71 ; 29.1 \%)$, cefuroxime $(n=44 ; 18 \%)$, cefixime $(n=27 ; 11 \%)$ were the most frequent prescribed antimicrobials (Table 2). Seventeen patients were hospitalized during sample obtaining, diagnosed as HAI and two of them had urine catheter in intensive care unit.

\begin{tabular}{|l|l|l|}
\hline \multicolumn{2}{|l|}{ Table 2: The prescribed antibiotics before the index positive urine culture (page 7) } \\
\hline $\begin{array}{l}\text { Antimicrobial } \\
\text { Agents }\end{array}$ & n & $\%$ \\
\hline Amoxicillin clavulanic acid & 71 & $29.1 \%$ \\
\hline Cefuroxime & 44 & $18 \%$ \\
\hline Cefixime & 27 & $11 \%$ \\
\hline Co-trimaxazole & 15 & $6.2 \%$ \\
\hline Ceftriaxone & 13 & $5.3 \%$ \\
\hline Claritromycine & 12 & $4.9 \%$ \\
\hline Cefdinir & 12 & $4.9 \%$ \\
\hline Nitrofurantoin & 10 & $4.1 \%$ \\
\hline Oseltamivir & 5 & $2 \%$ \\
\hline Metronidazole & 7 & $2.9 \%$ \\
\hline Sulbactam-Ampicillin & 5 & $2 \%$ \\
\hline Gentamicin & 4 & $1.6 \%$ \\
\hline Phosphomycine & 4 & $1.6 \%$ \\
\hline Penicillin & 3 & $1.2 \%$ \\
\hline Azithromycin & 3 & $1.2 \%$ \\
\hline Amikacin & 1 & $0.4 \%$ \\
\hline Others & 8 & $3.3 \%$ \\
\hline (Flucanazole, spiramycine, cefpodoxime, ciprofloxacin, cefazoline, cefalexine) \\
\hline
\end{tabular}

Dipstick tests were performed for 266 patients and $79.7 \%(n=212)$ of the results were compatible with laboratory UTI. Gram negative bacilli were the most common infectious agents and E.coli was leading ( $\mathrm{n}=$ $268 ; 78.4 \%)$. The rate of Gram positive agents was $6.4 \%$ $(\mathrm{n}=22)$ (Table 3). Antibiotic susceptibility tests showed low cephalosporine resistance unless ESBL is positive; however, ampicillin and co-trimoxazole seem to be inappropriate options for empiric treatment in our population. The rate of oxacillin resistance in Gram positive agents was $50 \%(n=11)$ and there was no vancomycin resistance in enterococci (Table 4).

Table 3: Distribution of infectious agents (page 8)

\begin{tabular}{|l|l|l|}
\hline & $\mathrm{n}$ & $\%$ \\
\hline Escherichia coli & 268 & 78.3 \\
\hline Gram (+) cocci & 22 & 6.5 \\
\hline Klebsiella strains (spp) & 19 & 5.6 \\
\hline Enterococcus spp & 12 & 3.6 \\
\hline Proteus mirabilis & 8 & 2.4 \\
\hline Pseudomonas aeruginosa & & \\
\hline Enterobacter spp & 4 & 1.2 \\
\hline Others (Acinetobacter spp, Citrobacter spp & 5 & 1.2 \\
\hline TOTAL & $\mathbf{3 4 2}$ & $\mathbf{1 0 0}$ \\
\hline
\end{tabular}


Table 4: Infectious agents and their antibigram results

\begin{tabular}{|c|c|c|c|c|c|c|c|c|c|c|c|c|c|c|c|c|c|}
\hline 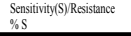 & Ampicillin & Amoxicillin Clavulanic scid & Piperacillin Tazbobctam & Cecturoxime & $\begin{array}{ll}\text { Cexoxitine } \\
\end{array}$ & Cefrixime & Ceftaridine & Cetrixizone & Etrapenem & Inipenam & Meropenem & \begin{tabular}{|lll} 
Amikacin & \\
\end{tabular} & Gentanicin & Cipoloxacine & 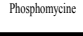 & Niroforantoin & 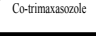 \\
\hline $\begin{array}{l}\text { E.coli } \\
n=268\end{array}$ & $\begin{array}{l}1181616 \\
47.0 \% \\
(251)\end{array}$ & $\begin{array}{l}14910101 \\
59.6 \% \% \\
(250)\end{array}$ & $\begin{array}{l}221226 \\
98.4 \% \\
(247)\end{array}$ & 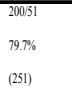 & $\begin{array}{l}23120 \\
9220 \% \\
(251)\end{array}$ & $\begin{array}{l}1955111 \\
83.7 \% \\
(2333)\end{array}$ & 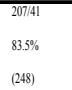 & $\begin{array}{l}211137 \\
85.1 \% \\
(248) \\
(248)\end{array}$ & $\begin{array}{l}2482 \\
2992 \% \\
(250) \\
(250)\end{array}$ & $\begin{array}{l}23111 \\
99.5 \% \\
(2322)\end{array}$ & $\begin{array}{l}24613 \\
98.8 \% \\
(2499)\end{array}$ & \begin{tabular}{|l|}
24566 \\
$97.6 \% 6$ \\
$(251)$
\end{tabular} & $\begin{array}{l}2341717 \\
932 \% \\
(251) \\
(251)\end{array}$ & \begin{tabular}{|l|}
214437 \\
$853 \% \%$ \\
$(2511)$
\end{tabular} & $\begin{array}{l}2303 \\
2987 \% \\
9233)\end{array}$ & $\begin{array}{l}23112 \\
99.1 \% \\
(233)\end{array}$ & $\begin{array}{l}184666 \\
73.6 \% \\
(250)\end{array}$ \\
\hline 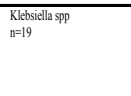 & $\begin{array}{l}1 / 18 \\
53 \% \\
(199)\end{array}$ & $\begin{array}{l}1109 \\
526 \% \% \\
\text { (19) }\end{array}$ & $\begin{array}{l}127 \\
127.1 \% \\
\text { (19) }\end{array}$ & $\begin{array}{l}1544 \\
189 \% \% \\
\text { (19) }\end{array}$ & $\begin{array}{l}172 \\
89.5 \% \\
\text { (19) }\end{array}$ & $\begin{array}{l}1313 \\
81.3 \% \\
\text { (16) }\end{array}$ & $\begin{array}{l}1544 \\
189 \% \% \\
\text { (19) }\end{array}$ & $\begin{array}{l}136 \\
\text { 6844\% } \\
\text { (19) }\end{array}$ & $\begin{array}{l}172 \\
89.9 \% \\
\text { (19) }\end{array}$ & $\begin{array}{l}1511 \\
99: 7 \% 6 \\
\text { (16) }\end{array}$ & $\begin{array}{l}190 \\
100 \% \\
\text { (19) }\end{array}$ & \begin{tabular}{|l|}
1900 \\
$100 \%$ \\
$(19)$ \\
$(19)$
\end{tabular} & $\begin{array}{l}1811 \\
94,7 \% \\
\text { (19) }\end{array}$ & \begin{tabular}{|l|}
172 \\
$89.5 \%$ \\
(19) \\
\end{tabular} & $\begin{array}{l}1333 \\
81.2 \% \\
\text { (16) }\end{array}$ & $\begin{array}{l}1511 \\
93.7 \% \\
\text { (16) }\end{array}$ & \begin{tabular}{|l|}
163 \\
$842 \%$ \\
(19)
\end{tabular} \\
\hline $\begin{array}{c}\text { Protes s sp } \\
n=8\end{array}$ & $\begin{array}{l}44 \\
5 \% \%\end{array}$ & $\begin{array}{l}62 \\
15 \% \%\end{array}$ & $\begin{array}{l}80 \\
100 \%\end{array}$ & $\begin{array}{l}62 \\
73 \% \\
68\end{array}$ & $\begin{array}{l}80 \\
100 \%\end{array}$ & $\begin{array}{l}80 \\
100 \%\end{array}$ & $\begin{array}{l}62 \\
75 \% \\
8 \%\end{array}$ & 53 & $\begin{array}{l}711 \\
879 \% \\
89\end{array}$ & $\begin{array}{l}80 \\
25 \%\end{array}$ & $\begin{array}{l}80 \\
100 \% \\
18 \%\end{array}$ & $\begin{array}{l}80 \\
100 \% \\
80 \%\end{array}$ & $\begin{array}{l}80 \\
100 \%\end{array}$ & $\begin{array}{l}80 \\
100 \% \\
80 \%\end{array}$ & $\begin{array}{l}80 \\
100 \%\end{array}$ & $\begin{array}{l}53 \\
625 \%\end{array}$ & $\begin{array}{l}53 \\
625 \% \\
\end{array}$ \\
\hline $\begin{array}{c}\substack{\text { Entintrococuls spp } \\
n=12} \\
\mathrm{n}=12\end{array}$ & $\begin{array}{l}\text { (1) } \\
\text { (10) }\end{array}$ & & & & & & & & & & & & & $\begin{array}{l}82 \\
80 \% \\
(10)\end{array}$ & & & $\begin{array}{l}773 \\
70 \% \\
(10)\end{array}$ \\
\hline $\begin{array}{l}\begin{array}{l}\text { Pseudomonas arengineosas } \\
n=4\end{array} \\
n=4\end{array}$ & & & $\begin{array}{l}30 \\
100 \% \\
\text { (3) }\end{array}$ & & & & $\begin{array}{l}30 \\
30 \% \\
100 \% \\
33)\end{array}$ & & & $\begin{array}{l}111 \\
50 \% 6 \\
(2)\end{array}$ & $\begin{array}{l}211 \\
66.6 \% \\
(3)\end{array}$ & $\begin{array}{l}211 \\
66.6 \% \\
\text { (3) } \\
\end{array}$ & $\begin{array}{l}211 \\
66.6 \% \\
\text { (3) } \\
\end{array}$ & $\begin{array}{ll}21 \\
66.6 \% \\
(3) \\
3\end{array}$ & & & \\
\hline $\begin{array}{c}\text { Staphlylococus spp } \\
\mathrm{n}=18\end{array}$ & & & & & & & & & & & & & $\begin{array}{l}1311 \\
92.2 \% \\
\text { (14) }\end{array}$ & \begin{tabular}{|l|}
131 \\
$92.8 \%$ \\
(14)
\end{tabular} & $\begin{array}{l}688 \\
428 \% \\
\text { (14) }\end{array}$ & & $\begin{array}{l}140 \\
100 \% \\
\text { (14) }\end{array}$ \\
\hline
\end{tabular}

ESBL positivity was detected in $58(17 \%)$ of the samples and these bacteria were susceptible to carbapenems, amikacin, phosphomycin, nitrofurantoin and piperacillin tazobactam (Table 5). Thirty-five $(60 \%)$ of the ESBL positive patients had previous antibiotic therapy history before present administration and antibiotic use was statistically significant for ESBL positivity $(p=0.01)$. ESBL was more frequent in females, however there was no statistical significance between genders $(\mathrm{p}=0.15)$. Most of the ESBL $(+)$ patients $(\mathrm{n}=48$; $82.8 \%$ ) were under 12 years old, the rate of positivity decreased with age, but this was not statistically significant, either $(\mathrm{p}=0.44)$. Also, UTI history within the three months had no significant effect on ESBL positivity $(\mathrm{p}=0.14)$. Only one ESBL $(+)$ patient had HAI, all others were evaluated as CA-UTI. After diagnosis, $18 \mathrm{pa}-$ tients were hospitalized for UTI treatment. Among 58 ESBL $(+)$ patients only six were hospitalized and 4 were treated with carbapenems and 2 with amikacin. The rest of the patients were treated with nitrofurantoin $(n=34)$, intramuscular amikacin $(n=5)$ and gentamicin $(n=9)$ in the outpatient settings. ESBL positivity was not a criterion for hospitalization $(p=0.11)$

Table 5: Antibiogram susceptibility of the ESBL(+) agents

\begin{tabular}{|c|c|c|c|c|c|c|c|c|c|c|c|c|c|c|c|c|c|}
\hline 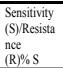 & \begin{tabular}{|c|} 
Ampicillin \\
\end{tabular} & $\begin{array}{l}\begin{array}{l}\text { Amoxicillin } \\
\text { Clauvalaic } \\
\text { Acid }\end{array} \\
\end{array}$ & $\begin{array}{l}\text { Piperacillin } \\
\text { Tazobactam }\end{array}$ & \begin{tabular}{|l|} 
Ceffuroxime \\
\end{tabular} & $\begin{array}{l}\text { Cefoxitine } \\
\end{array}$ & $\begin{array}{l}\text { Cefixime } \\
\text {. }\end{array}$ & $\begin{array}{l}\text { Ceftazidime } \\
\end{array}$ & Ceffriaxone & $\begin{array}{l}\text { Ertapencm } \\
\end{array}$ & $\begin{array}{l}\text { Imipenem } \\
\end{array}$ & Meropenem & $\begin{array}{l}\text { Amikacin } \\
\end{array}$ & Gentamicin & Ciprofloxacine & Phosphomycine & Nitrofirantoin & $\begin{array}{l}\text { Co- } \\
\text { trimaxasoole }\end{array}$ \\
\hline $\begin{array}{l}\substack{E \text { Ecoli } \\
\mathrm{n}=50} \\
=\end{array}$ & $\begin{array}{l}244 \\
4.3 \% \\
(46)\end{array}$ & $\begin{array}{l}2234 \\
26 \% \\
(46)\end{array}$ & $\begin{array}{l}3977 \\
84.7 \\
(46)\end{array}$ & \begin{tabular}{|l|}
7339 \\
$15.2 \%$ \\
$(46)$
\end{tabular} & $\begin{array}{l}32 / 14 \\
69.5 \% \\
(46)\end{array}$ & $\begin{array}{l}9 / 35 \\
20.4 \% \\
(44)\end{array}$ & $\begin{array}{l}638 \\
13.6 \% \\
(44)\end{array}$ & $\begin{array}{l}1333 \\
28.2 \% \\
(46)\end{array}$ & $\begin{array}{l}45 / 1 \\
97.8 \% \\
(46)\end{array}$ & $\begin{array}{l}43 / 11 \\
97.7 \% \\
(44)\end{array}$ & $\begin{array}{l}45 / 11 \\
97.8 \% \\
(46)\end{array}$ & $\begin{array}{l}4214 \\
91.3 \% \\
(46)\end{array}$ & $\begin{array}{l}388.8 \\
82.6 \% \\
(46)\end{array}$ & $\begin{array}{l}27719 \\
58.7 \% \\
(46)\end{array}$ & $\begin{array}{l}43311 \\
97.7 \% \\
(44)\end{array}$ & $\begin{array}{l}3311 \\
97.7 \% \\
(44)\end{array}$ & $\begin{array}{l}232323 \\
50 \% \\
(46)\end{array}$ \\
\hline 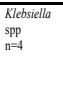 & $\begin{array}{l}04 \\
0 \\
\text { (4) }\end{array}$ & $\begin{array}{l}0 / 4 \\
0 \\
\text { (4) }\end{array}$ & $\begin{array}{l}1 / 3 \\
25 \% \\
\text { (4) }\end{array}$ & $\begin{array}{l}1 / 3 \\
25 \% \\
\text { (4) }\end{array}$ & $\begin{array}{l}4,00 \\
100 \% \\
\text { (4) }\end{array}$ & $\begin{array}{l}1 / 2 \\
66.6 \% \\
\text { (3) }\end{array}$ & $\begin{array}{l}1 / 3 \\
25 \% \\
\text { (4) }\end{array}$ & $\begin{array}{l}0 / 4 \\
0 \\
(4)\end{array}$ & $\begin{array}{l}400 \\
100 \% \\
(4)\end{array}$ & $\begin{array}{l}3 / 0 \\
100 \% \\
\text { (3) }\end{array}$ & $\begin{array}{l}4 / 0 \\
100 \% \\
(4)\end{array}$ & $\begin{array}{l}4 / 0 \\
100 \% \\
(4)\end{array}$ & $\begin{array}{l}3 / 1 \\
75 \% \\
\text { (4) }\end{array}$ & $\begin{array}{l}3 / 1 \\
75 \% \\
\text { (4) }\end{array}$ & $\begin{array}{l}300 \\
100 \% \\
\text { (3) }\end{array}$ & $\begin{array}{l}2 / 1 \\
66.6 \% \\
\text { (3) }\end{array}$ & $\begin{array}{l}2 / 2 \\
50 \% \\
\text { (4) }\end{array}$ \\
\hline 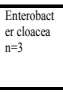 & $\begin{array}{l}0 / 3 \\
0 \\
\text { (3) } \\
\end{array}$ & $\begin{array}{l}0 / 3 \\
0 \\
(3) \\
\end{array}$ & $\begin{array}{l}0 / 2 \\
0 \\
(2) \\
\end{array}$ & $\begin{array}{l}0 / 3 \\
0 \\
(3) \\
\end{array}$ & $\begin{array}{l}0 / 3 \\
0 \\
\text { (3) } \\
\end{array}$ & $\begin{array}{l}0 / 2 \\
0 \\
(2) \\
\end{array}$ & $\begin{array}{l}0 / 3 \\
0 \\
(3) \\
\end{array}$ & $\begin{array}{l}0 / 3 \\
0 \\
(3) \\
\end{array}$ & $\begin{array}{l}30 \\
100 \% \\
\text { (3) } \\
\end{array}$ & $\begin{array}{l}20 \\
100 \% \\
\text { (2) } \\
\end{array}$ & $\begin{array}{l}30 \\
100 \% \\
\text { (3) }\end{array}$ & $\begin{array}{l}30 \\
100 \% \\
(3) \\
\end{array}$ & $\begin{array}{l}300 \\
100 \% \\
\text { (3) }\end{array}$ & $\begin{array}{l}30 \\
100 \% \\
\text { (3) }\end{array}$ & $\begin{array}{l}1 / 1 \\
50 \% \\
(2) \\
\end{array}$ & $\begin{array}{l}20 \\
100 \% \\
\text { (2) }\end{array}$ & $\begin{array}{l}30 \\
100 \% \\
\text { (3) }\end{array}$ \\
\hline 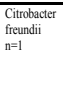 & $\begin{array}{l}0 / 1 \\
0 \\
\text { (1) }\end{array}$ & $\begin{array}{l}0 / 1 \\
0 \\
\text { (1) }\end{array}$ & $\begin{array}{l}100 \\
100 \% \\
\text { (1) }\end{array}$ & $\begin{array}{l}0,1 \\
0 \\
\text { (1) }\end{array}$ & $\begin{array}{l}1 / 0 \\
100 \% \\
\text { (1) }\end{array}$ & & $\begin{array}{l}0,1 \\
0 \\
\text { (1) }\end{array}$ & $\begin{array}{l}0 / 1 \\
0 \\
\text { (1) }\end{array}$ & $\begin{array}{l}10 \\
100 \% \\
\text { (1) }\end{array}$ & & $\begin{array}{l}1 / 0 \\
100 \% \\
\text { (1) }\end{array}$ & $\begin{array}{l}10 \\
100 \% \\
\text { (1) }\end{array}$ & $\begin{array}{l}1 / 0 \\
100 \% \\
\text { (1) }\end{array}$ & $\begin{array}{l}0 / 1 \\
0 \\
\text { (1) }\end{array}$ & & & $\begin{array}{l}1 / 0 \\
100 \% \\
\text { (1) }\end{array}$ \\
\hline
\end{tabular}

\section{Discussion}

Urinary tract infections are common problems in the pediatric population. The diagnosis and treatment may be challenging sometimes, but morbidity and mortality can be inevitable due to short and long- time outcomes, unless it is well treated ${ }^{6-8}$. Empirical treatment strategies are important because studies to detect the infectious agents and their antimicrobial susceptibility take time. The agents and their antibiotic resistance profile may vary between different areas, age groups, clinical status and may alter within time $e^{21,22}$. In this retrospectively designed study we focused on culture positive UTI, related risk factors and treatment choices for this common problem.

During a one year period 3640 urine specimens from toilet trained children over 5 years old were evaluated and 342 had significant growth; the rate of culture pos- 
itivity was $9.6 \%$. UTI prevalence is high throughout the world, it was reported as 16\% from Nepal, similar to South- East Asia, but lower in the USA and Iran 9\%, $7.87 \%$ respectively ${ }^{9}, 23,24$. The rates of UTI may be different in different areas. According to the clinical and sociodemographic features, results may vary. Our study group consisted of children who were able to give clean catch specimen and with no risk factors related to recurrent infection. This may be the reason of lower UTI prevalence in this study group. The ratio between females and males in the study population was 2.3 and 9.3 within the culture positive group. The high rate in girls is thought to be related with the anatomic structure. Low rate in boys may be the result of circumcision as it prevents the entrance of microorganisms to the urinary tract from the prepuce ${ }^{25}$. In Turkey most of the boys have circumcision before age 6 years due to regional rules and tradition. The rate of UTI may vary between genders due to age ${ }^{9}$, however age and gender had no statistical significance in our study although infection rate decreased with age and it was high in females.

In children, UTI presents with non-specific symptoms; especially in young children as they cannot express themselves in words. Fever, irritability, anorexia, abdominal pain, nausea, vomiting, urinary symptoms such as dysuria, polyuria, vesical tenesmus, urgency, incontinence are the common problems at admission., ${ }^{9}{ }^{26}$. Fever, poor feeding, irritability were the most frequent symptoms in younger children as reported in many studies. The members of this study group were at the age that they can express themselves and abdominal pain and/or dysuria were the most common reasons for admission. The symptoms directed the practitioners to urine tests and positivity/ negativity ratio of dipstick tests were compatible with positiveculture results (212/44).

The most common detected microorganism causing UTI was E.coli in our study group as it is in many reports $^{9,12}$. E.coli originate from the faecal flora, spread to the urinary tract and become an infectious agent irrespective to age, gender or season26.Gram negative bacilli are responsible for majority of UTI, but recently Gram positive microorganisms have been also reported as it is in our study?.

Multi drug resistance (MDR) to antimicrobial agents is a global public health problem. One of the mechanisms of resistance is to produce ESBL which makes the treatment unresponsive to first choice antibiotics such as cephalosporins for UTI. This increases the morbidity of infection and health care costs via long hospital stay or maltreatment complications. The rate of third generation cephalosporin susceptibility was over $85 \%$ in ESBL negative agents. In this study, the rate of ESBL positivity was $17 \%$, lower than many countries. The susceptibility profile consisted of carbapenems (98-100\%), phosphmycine (98-100\%), aminoglycosides (91-100\%); compatible with the literature. Nitrofurantoin susceptibility was approximately $98 \%$ to E.coli, but $66.6 \%$ to Klebsiella spp. Carbapenems have the lowest resistance rate for ESBL $(+)$ agents among other antibiotics ${ }^{27}$. However they are expensive and applied by infusion which makes hospitalization necessary; they are good choices for severe infections or when resistance exists to other antimicrobials ${ }^{28}$. Also they are under cover of the social insurance institution when the patient is hospitalized and one cannot obtain these kind of drugs without formal procedures in our country. In this study 4 patients were treated with meropenem at the hospital. Faropenem and Tebipenem are oral forms of carbapenems, but they are not in the market of our country and experience in the pediatric population is limited ${ }^{29}$.

The role of narrow spectrum antibiotics is popular and nitrofurantoin is a good option with low resistance rate, for E.coli. It must be administered four times a day and this makes the patient compliance difficult, but it is applied orally ${ }^{30}$. When UTI is uncomplicated nitrofurantoin seems to be a suitable drug for our population with resistance rate as $0.9 \%$ for E.coli. The rate of phosphomycine susceptibility in ESBL $(+)$ agents was approximately 100\% in this study group. Phosphomycine is applied once orally, this provides excellent patient compliance, however experience is limited in children30. Amikacin is one of the most popular agents in pediatric UTI treatment practice with its long experience history. It was a good choice against Klebsiella spp in this group with a susceptibility rate of $100 \%$. A recent study recommends once-daily intramuscular amikacin as an alternative option for outpatient treatment of community-acquired lower UTIs, but the patients must be monitored for nephrotoxicity and ototoxicity ${ }^{14}$. Co-trimoxazole is another choice in USA, but in Turkey resistance rate is high ${ }^{31,32}$. An antibiotic should not be recommended when the resistance rate is over $20 \%$ for empirical treatment ${ }^{31}$. The rate of resistance was approximately $25 \%$ in our study group, revealing that it is not a suitable choice for our population. Quinolones are other alternative options for the adult population, but they are not recommended for children because of osteoarticular side effects and increasing resistance rate unless UTI is complicated ${ }^{31,33}$. Piperacillin tazobactam may be considered as an alternative option in our population against E.coli, varying from other reports? 
In this study group 18 patients were hospitalized for UTI treatment. Six of them were infected by ESBL microorganisms. This showed that the clinical status (poor feeding, vomiting, drug intolerance, and lassitude) of the patient is the significant parameter that effects the clinician's decision about hospitalization, not the microorganisms. Among $58 \mathrm{ESBL}(+)$ patients just six were hospitalized and 4 were treated with carbapenems and 2 with amikacin. The rest of the patients were treated with nitrofurantoin $(n=34)$, intramuscular amikacin ( $n=$ $5)$ and gentamicin $(n=9)$ in the outpatient settings.

Risk factors for ESBL positivity were defined as "having urogenital anomalies or neuromuscular problems related with non-optimal urine drainage, clean intermittent catheterization necessity, $<1$ year-old age, having high UTI recurrence rate, long duration of prophylaxis, use of cephalosporin for prophylaxis, hospitalization within previous 3 months" by Kizilca et al ${ }^{17}$. Our study group consisted of $>5$ years old children having no risk factors defined by Kizilca et al., but these must be always in consideration while managing UTI. The only risk factor we could define was antibiotic intake within the preceding three months before the culture test. Gender, age, season, UTI history of previous UTI had no statistically significant effect on ESBL positivity.

\section{Conclusion}

We recommend that preventing UTI is easier than treating the infection. Keeping the perineum clean, not using wet wipes for cleaning, wiping without contaminating anal region, keeping away ointments containing steroids for perianal dermatologic care are supportive methods for preventing UTI. Also circumcision should be recommended for boys. Third generation cephalosporins are still good options for UTI treatment in patients having no risk factors defined above. The resistance rate is under $20 \%$ for ceftriaxone, cefixime, cefoxitin and ESBL positivity rate is $17 \%$ in our study group. However, these alarming rates will lead to increasing resistance if antibiotic abuse carries on. Antibiotics should be prescribed for necessary indications with right dose per kilograms and enough duration of treatment. Co- trimoxazole and amoxicillin clavulanate seem to be bad options for UTI management for our population. If there are risk factors as Kizılca et al. defined or antibiotic intake within three months, ESBL positivity must be considered. The patients should be hospitalized if he/she is in bad clinical condition to provide supportive treatment and oral intake. In vivo response of third generation cephalosporines to ESBL $(+)$ E.coli may be better than in vitro tests. This situa- tion can be explained by the higher concentrations of antimicrobials in the urine ${ }^{34}$. When there is successful clinical response to the initiated antibiotic (resolution of fever and other symptoms, decreasing trend of acute phase reactants (leukocytes, C- reactive protein) and urine leukocyte count, sterile urine culture at $<72$ hours), the same agent can be continued even the result of the urine culture is an ESBL $(+)$ agent $^{35}$. Amikacin, gentamicin, carbapenems, piperacillin tazobactam can be ordered for unresponsive and selective cases. Nitrofurantoin, amikacin, gentamicin can be good options for outpatient settings if patient compliance is good. Patients should be monitored for nephrotoxicity and ototoxicity during aminoglycosides therapy. More studies are needed about efficiency of phosphomycin and oral carbapenems in children. Also, phosphomycin susceptibility must be evaluated via agar dilution methods for more accurate results ${ }^{20}$. Good clinical practice and well organized regional surveillance programs are necessary to update the treatment guidelines.

This report has great limitations as it is a retrospective study based on hospital records. The results cannot be generalized to the whole population as only the children visiting our hospital were included in the study. Also, younger children having no toilet training and having defects related to recurrent UTI, the most affected populations from UTI, were excluded. There is lack of data about clinical outcome of the cases. Also, the faecal flora of the cases were not considered although ESBL positivity was reported. We recommend that multicenter surveillance including all pediatric age groups should be conducted in the future with longer clinical follow up.

\section{Funding and conflict of interest declaration}

This study has no funding and the authors declare no conflict of interest or potential conflict of interest.

\section{References}

1. Shaikh N, Morone NE, Bost JE, Farrell MH. Prevalence of urinary tract infection in childhood: a meta-analysis. Pediatr Infect Dis J. 2008; 27(4):302-8. PMID: 18316994; doi: 10.1097/INF.0b013e31815e4122

2. Downing H, Thomas-Jones E, Gal M, Waldron CA, Sterne J, Hollingworth W, et al. The diagnosis of urinary tract infections in young children (DUTY): protocol for a diagnostic and prospective observational study to derive and validate a clinical algorithm for the diagnosis of UTI in children presenting to primary care with an acute illness. BMC Infect Dis. 2012;12:158. PMID: 22812651; doi:10.1186/1471-2334-12-158 
3. Desai DJ, Gilbert B, McBride CA. Paediatric urinary tract infections: diagnosis and treatment. Aust Fam Physician. 2016;45(8):558-563 PMID: 27610444

4. Stein R, Dogan HS, Hoebeke P Kočvara R, Nijman RJ, Radmayr C, et al. Urinary tract infections in children: EAU/ESPU guidelines. Eur Urol. 2015;67 (3):546-558. PMID: 25477258 DOI: 10.1016/j.eururo.2014.11.007

5. World Health Organization (WHO). Prevention of Hospital-Acquired Infections. 2nd edn. Geneva: WHO; Available from: www.who.int/csr/resources/publications/whocdscsreph200212.pdf，2002. (Accessed in June, 2019).

6. Zorc JJ, Kiddoo DA, Shaw KN. Diagnosis and management of pediatric urinary tract infections. Clin $\mathrm{Mi}$ crobiol Rev. 2005;18(2):417-422 PMID: 15831830; DOI: 10.1128/CMR.18.2.417-422.2005

7. Montini G, Tullus K, Hewitt I. Febrile urinary tract infections in children. N Engl J Med. 2011;365(3):239_ 250. PMID: 21774712 DOI: $10.1056 /$ NEJMra1007755 8. Vijayakumar M, Kanitkar M, Nammalwar BR, Bagga A. Revised statement on management of urinary tract infections. Indian Pediatr. 2011;48(9):709-17. PMID: 21992903

9. Shrestha LB, Baral R, Poudel P, Khanal B. Clinical, etiological and antimicrobial susceptibility profile of pediatric urinary tract infections in a tertiary care hospital of Nepal. BMC Pediatrics 2019(1);19:36. PMID: 30696410; DOI: 10.1186/s12887-019-1410-1

10. Nagaraj S, Kalal BS, Kamath N, Muralidharan S. Microbiological and antimicrobial profile of pathogens associated with pediatric urinary tract infection: A one year retrospective study from a tertiary care teaching hospital. National Journal of Laboratory Medicine. 2014;3(1):4-7. DOI: NJLM/2014/7651:2001

11. Mishra OP, Abhinay A, Prasad R. Urinary infections in children. Indian J Pediatr. 2013;80(10):838-843. PMID: 23881478 DOI: $10.1007 / s 12098-013-1118-4$

12. Kalal BS, Patel R. Microbiological and Antimicrobial Profile of Urinary Tract Infection in Children From A Teaching Hospital in South India. J Pediatr Inf 2017; 11: 19-22. DOI: $10.5578 /$ ced.50691

13. Pitout JD, Laupland KB. Extended-spectrum beta-lactamase-producing Enterobacteriaceae: an emerging public-health concern. Lancet Infect Dis. 2008;8(3):159-166. PMID: 18291338 DOI: 10.1016/ S1473-3099(08)70041-0

14. Polat M, Kara SS. Once-daily intramuscular amikacin for .outpatient treatment of lower urinary tract infections caused by extended-spectrum b-lactamase-producing Escherichia coli in children. Infection and Drug Resistance 2017;10(1):393-399 PMID: 29138582; DOI: 10.2147/IDR.S148703
15. Cantón R, Akóva M, Carmeli Y, Giske CG, Glupczynski Y, Gniadkowski M, et al. European Network on Carbapenemases. Rapid evolution and spread of carbapenemases among Enterobacteriaceae in Europe. Clin Microbiol Infect. 2012;18(5):413-431. PMID: 22507109 DOI: 10.1111/j.1469-0691.2012.03821.x

16. Robinson JL, Le Saux N. Management of urinary tract infections in children in an era of increasing antimicrobial resistance. Expert Rev Anti Infect Ther. 2016;14(9):809-816. https://doi.org/10.1080/1478721 0.2016 .1206816

17. Kizilca O, Siraneci R, Yilmaz A, Hatipoglu N, Ozturk E, Kiyak A, et al. Risk factors for community-acquired urinary tract infection caused by ESBL-producing bacteria in children. Pediatr Int. 2012;54(6):858-862. https://doi.org/10.1111/j.1442-200X.2012.03709.x

18. Lukac PJ, Bonomo RA, Logan LK. Extended-spectrum B-lactamase-producing Enterobacteriaceae in children: Old foe, emerging threat. Clin Infect Dis. 2015;60(9):1389-1397. PMID: 25595742; DOI: $10.1093 / \mathrm{cid} / \mathrm{civ} 020$

19. Hooton TM, Bradley SF, Cardenas DD, Colgan R, Geerlings SE, Rice JC, et al. Diagnosis, prevention, and treatment of catheter-associated urinary tract infection in adults: 2009 international clinical practice guidelines from the Infectious Diseases Society of America. Clin Infect Dis. 2010;50(5):625-663. https://doi. org/10.1086/650482

20. The European Committee on Antimicrobial Susceptibility Testing. Breakpoint tables for interpretation of MICs and zone diameters. Version 5.0, 2015. Available from: PDFs/EUCAST_files/Breakpoint_tables/v_5.0_Breakpoint_Table_01. pdf. Accessed April 25, 2019.

21. Gupta P, Mandal J, Krishnamurthy S, Barathi D, Pandit N. Profile of urinary tract infections in paediatric patients. Indian J Med Res [serial online] 2015 [cited 2019 Oct 16];141:473-7. Available from: http://www. ijmr.org.in/text.asp?2015/141/4/473/159299

22. Akhtar MS, Mohsin N, Zahak A, Ain MR, Pillai PK, Kapur P, et al. Antimicrobial sensitivity pattern of bacterial pathogens in urinary tract infections in South Delhi, India. Rev Recent Clin Trials. 2014; 9:271-275. PMID: 25441943

23. Zorc JJ, Levine DA, Platt SL, Dayan PS, Macias CG, Krief W, et al. Clinical and demographic factors associated with urinary tract infection in young febrile infants. Pediatrics. 2005(3); 116:644-648. PMID: 16140703 DOI: $10.1542 /$ peds.2004-1825

24. Ammenti A, Cataldi L, Chimenz R, Fanos V, La Manna A, Marra G, et al. Febrile urinary tract infections in young children: recommendations for 
the diagnosis, treatment and follow-up. Acta Paediatr. 2012;101(5):451-457. PMID: 22122295 DOI: 10.1111/j.1651-2227.2011.02549.x

25. Laway MA, Wani ML, Patnaik R, Kakru D, Ismail S, Shera AH, et al. Does circumcision alter the periurethral uropathogenic bacterial flora. Afr J Paediatr Surg. 2012;9(2):109-112. PMID: 22878757 DOI: 10.4103/0189-6725.99394

26. Korbel L, Howell M, Spencer JD. The clinical diagnosis and management of urinary tract infections in children and adolescents. Paediatr Int Child Health. 2017;37(4):273-279. https://doi.org/10.1080/204690 47.2017.1382046

27. Tosun İstanbullu A, Demirci M, Yılmaz M, Şen H, Sirekbasan L, Şaylan Gözün E, Gökçeağaçlı C, Şengil AZ. Antimicrobial Resistance Rates of Escherichia coli and Klebsiella pneumoniae Strains Isolated from Urinary Tract Infections. ANKEM Derg 2016;30(1):1-6 doi: 10.5222/ankem.2016.001

28. Nivesvivat T, Piyaraj P, Thunyarn S, Watanaveeradej V, Suwanpakdee D. Clinical epidemiology, risk factors and treatment outcomes of extended spectrum beta lactamase producing Enterobacteriaceae bacteremia among children in a Tertiary Care Hospital, Bangkok, Thailand. BMC Res Notes 2018; 11:624. https://doi. org/10.1186/s13104-018-3729-3

29. Livermore DM. Has the era of untreatable infections arrived? I Antimicrob Chemother 2009;64 (S1):2936. PMID: 19675016 DOI: $10.1093 / \mathrm{jac} / \mathrm{dkp} 255$

30. Alpay Y, Yavuz MT, Aslan T, Büyükzengin B. Can Oral Antibiotics Be an Alternative to Carbapenems in The Treatment of Non-Complicated Urinary Tract Infections Caused by Extended Spectrum Beta-lactamase
Positive Escherichia coli? ANKEM Derg 2017;31(3):8591. doi: 10.5222/ankem.2017.085

31. Gupta K, Hooton TM, Naber K, Wullt B, Colgan R, Miller LG, et al. International clinical practice guidelines for the treatment of acute uncomplicated cystitis and pyelonephritis in women: a 2010 update by the Infectious Diseases Society of America and the European Society for Microbiology and Infectious Diseases. Clin Infect Dis 2011;52(5):103-120. PMID: 21292654 DOI: $10.1093 / \mathrm{cid} / \mathrm{ciq} 257$

32. Çalışkan E, Dede A, Altınöz Aytar A, Biten Güven G, Kaş E. The Evaluation of Resistance Rates to Various Antibiotics and Extended Spectrum Beta-lactamase Positivity of Esherichia coli and Klebsiella spp. Strains Isolated from Outpatients with Urinary Tract Infection. ANKEM Derg 2015;29(2):47-53. doi:10.5222/ ankem.2015.047

33. American Academy of Pediatrics, Committee on Infectious Diseases. The use of systemic and topical fluoroquinolones. Pediatrics. 2011;128(4):1034-1045. PMID: 21949152 DOI: 10.1542/peds.2011-1496

34. Peco-Antic A, Paripovic D, Buljugic S, Spasojević-Dimitrijeva $B$, Cvetković $M$, Laban-Nestorović $S$, et al. In vivo susceptibility of ESBL producing Escherichia coli to ceftriaxone in children with acute pyelonephritis. Srp Arb Celok Lek. 2012;140(5-6):321-5. PMID: 22826985

35. Balasubramanian S, Kuppuswamy D, Padmanabhan S, Chandramohan V, Amperayani S. Extended-spectrum Beta-lactamase-producing Community-acquired Urinary Tract Infections in Children: Chart Review of Risk Factors. J Glob Infect Dis. 2018; 10(4): 222-225. PMID: 30581265; doi: 10.4103/0974-777X.246391 